\title{
Abstract
}

\section{Prevalence of Obesity Among Adults in Jordan: National Survey}

\author{
Ansam Al Hadidi, MD \\ Jordanian Ministry of Health, Amman, Jordan
}

\section{Corresponding Author:}

Ansam Al Hadidi, MD

Jordanian Ministry of Health

ALhashimi Alshamaly

Basman Area, Hay Naifah

Amman

Jordan

Phone: 962770401185

Email: ansamhadidi22@gamil.com

\section{Abstract}

Background: Obesity is a national and global public health problem in terms of morbidity, mortality, and economic burden. In $2014,5 \%$ of deaths worldwide were attributable to obesity, with an estimated economic impact of $2.8 \%$ of the global gross domestic product. A survey in 2008 showed a high prevalence of overweight and obesity among adults in Jordan.

Objective: This study aimed to determine the prevalence of obesity, assess its trends, and identify the factors and comorbidities associated with this condition.

Methods: A multipurpose national household survey was conducted among Jordanian adults over 4 months in 2017 . Data were collected using a structured validated questionnaire. Obesity was defined according to the International Diabetes Federation criteria.

Results: This study included 4056 adults (1193 men, 29.4\%; 2863 women, 70.6\%). Their age ranged from 18 to 90 years, with a mean age of 43.8 (SD 14.2) years. According to the International Diabetes Federation criteria, the age-standardized prevalence of obesity was $60.4 \%$ in men and $75.6 \%$ in women. After adjusting for age, the odds of obesity in 2017 were twice the odds in 2008 in men (odds ratio [OR] 1.98) and women (OR 1.96). In the multivariate analysis, age, place of living, and marital status were significantly associated with obesity in men and women. Obesity was significantly associated with increased odds of diabetes mellitus (OR 2.1 for men; OR 2.9 for women), hypertension (OR 2.4 for men; OR 2.5 for women), elevated triglyceride levels (OR 2.5 for men; OR 4.2 for women), and low high-density lipoprotein levels (OR 2.2 for men; OR 2.1 for women) after adjusting for age.

Conclusions: The prevalence of obesity is high in Jordan, and this condition is associated with other metabolic abnormalities. Well-defined programs to manage, control, and prevent obesity as well as intersectoral action are urgently required to reverse current trends in Jordan.

(iproc 2022;8(1):e36398) doi: 10.2196/36398

\section{KEYWORDS}

obesity; survey; waist circumference; metabolic abnormality

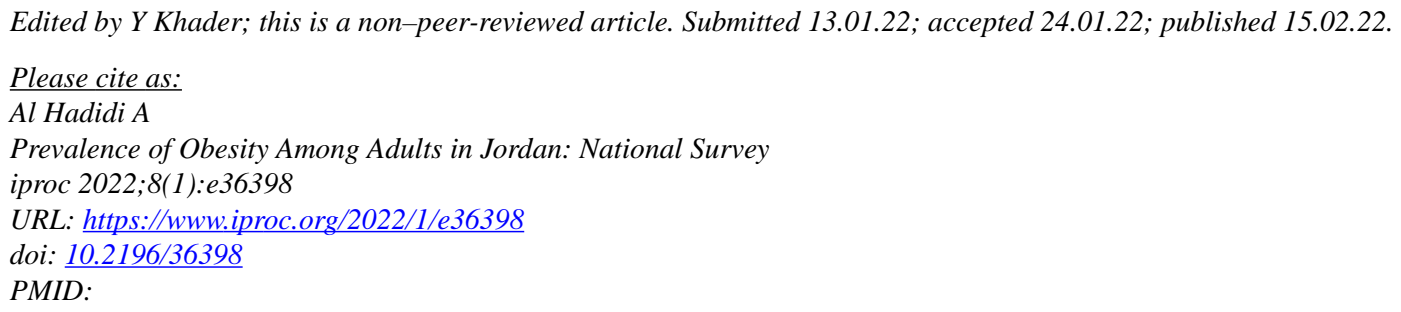


(C)Ansam Al Hadidi. Originally published in Iproceedings (https://www.iproc.org), 15.02.2022. This is an open-access article distributed under the terms of the Creative Commons Attribution License (https://creativecommons.org/licenses/by/4.0/), which permits unrestricted use, distribution, and reproduction in any medium, provided the original work, first published in Iproceedings, is properly cited. The complete bibliographic information, a link to the original publication on https://www.iproc.org/, as well as this copyright and license information must be included. 